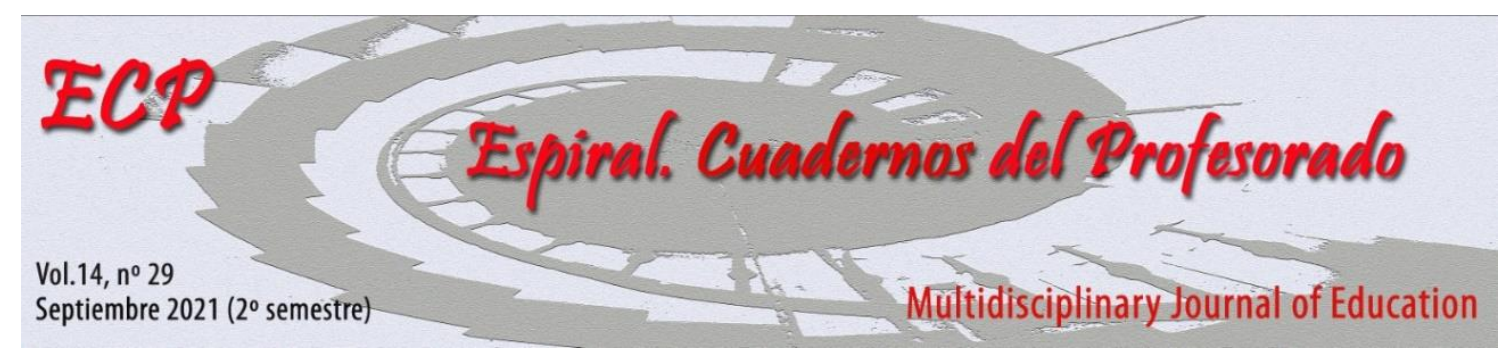

\title{
Discursos educativos en torno a lo digital, en Portugal: ¿qué lugar para la creación (literaria)?
}

\section{Educational discourses on digital, in Portugal: what place to (literary) creation?}

\author{
Ângela Balça y Paulo costa \\ Universidade de Évora / CIEC, Portugal
}

\begin{abstract}
Resumen
$\mathrm{Al}$ identificar un conjunto de textos que, por su génesis y naturaleza, pueden configurarse como discursos educativos determinantes, en Portugal, se intentó identificar, mediante el uso del análisis documental, cómo la presencia de lo digital, especialmente cuando se articula con la creación (y la creación literaria en particular) se materializa en estos. Para ello, seleccionamos el Perfil de los Alumnos a la Salida de la Educación Obligatoria y los documentos elaborados en el marco de la Estrategia Nacional de Educación para la Ciudadanía, el Plan Nacional de Lectura y la Red de Bibliotecas Escolares. Concluimos que existe un alto grado de compatibilidad entre los distintos documentos analizados, especialmente en lo que se refiere a la importancia que, en el mundo contemporáneo, tiene la tecnología y, en particular el digital asumen. Asimismo, todos los documentos están alineados con la necesidad de formar estudiantes / ciudadanos responsables / críticos que construyan un futuro sostenible. En este proceso, se asume que la creatividad, la creación, la sensibilidad estética juegan un papel sumamente relevante. Si bien, de manera explícita, la relación entre digital y creación solo aparece de manera tenue, consideramos que los documentos dejan un margen de maniobra positivo a los involucrados en los procesos, para que la sinergia entre tecnología digital y proceso de creación sea posible.
\end{abstract}

Palabras clave: discurso educativo; ciudadanía; digital; creación.

\begin{abstract}
When identifying a set of texts that, due to their genesis and nature, can be configured as determining educational discourses, in Portugal, we intended to identify, through unobtrusive data analysis, how the presence of the digital, especially when articulated with creation (and literary creation in particular) is materialized in those discourses. To do this, we selected the Profile of Students Leaving Compulsory Education and the documents prepared within the framework of the National Strategy for Citizenship Education, the National Reading Plan and the Network of School Libraries. We concluded that there is a high degree of compatibility between the different documents analysed, especially with regard to the importance that, in the contemporary world, technology and, in particular, digital technology assume. Likewise, all the documents are aligned with the need to prepare responsible/critical students/citizens able to build a sustainable future. In this process, it is assumed that creativity, creation, and aesthetic sensitivity play an extremely relevant role. Although, explicitly, the relationship between digital and creation appears only tenuous, we consider that the documents allow a positive leeway for those involved in the processes, so that the synergy between digital technology and creation process is possible.
\end{abstract}

Keywords: educational discourse; citizenship; digital; creation.

Fecha de recepción: 09/02/2021

Fecha de aceptación: 07/07/2021 


\section{Introducción}

Este texto se analiza la presencia de lo digital en el discurso pedagógico en Portugal y se reflexiona sobre las posibles implicaciones de esa misma presencia. Actualmente, el surgimiento y progresivo aumento de la importancia de lo digital en la vida de la sociedad y los ciudadanos es una evidencia, de hecho, no existen áreas que no estén marcadas y conectadas por el espacio digital (Morgado, 2019). La escuela no puede ser una excepción en esta realidad y cada vez más la sociedad entiende el contexto escolar como una instancia de formación de los jóvenes para el mundo digital. Si en un principio nos parece que los más jóvenes son los que, en algunos contextos, se han denominado nativos digitales, estudios posteriores revelan que, a pesar de la enorme facilidad en el manejo de herramientas y dispositivos digitales, estos jóvenes presentan grandes brechas para afrontar un mundo mucho más complejo.

¿Tienen estos jóvenes un buen dominio de la competencia lectora y digital? ¿Conocen los procedimientos de seguridad que deben observar cuando se encuentran en el espacio digital? ¿Son estos jóvenes capaces de salvaguardar su salud y bienestar en un mundo digital que puede contribuir a ciertas adiciones? ¿Conocen las reglas fundamentales para comportamientos e implicaciones adecuadas y de calidad en la web? ¿Será que, además de los aspectos intrínsecamente instrumentales que el predominio de lo digital hace emerger como retos esenciales, la escuela perderá el foco en su vocación última: formar personas?

Estas son solo algunas de las preguntas que surgen y que visibilizan la necesidad de la formación de los más jóvenes no solo para el dominio de alfabetizaciones múltiples, sino también para la e-ciudadanía o ciudadanía digital. Si leemos el estudio de Rosário (2021, en prensa) nos damos cuenta de que existen debilidades bastante serias en la formación de habilidades de alfabetización de estos jóvenes y que el mundo digital no está contribuyendo a llenar este hueco; la generación más joven presenta

Baixíssimos consumos, por parte dos jovens, da imprensa tradicional escrita (jornais e revistas), sem que, no entanto, isso signifique uma transferência estrita (e crescente) para os formatos da imprensa digital - conclusão que também situa as amostras trabalhadas na moldura interna e além-fronteiras; Fraca possibilidade de formação de uma classe leitora (juvenil) (Rosário, 2021, en prensa) ${ }^{1}$

La presentación preliminar de los resultados del estudio de Mata y Neves (2020), sobre las prácticas lectoras de estudiantes de educación primaria y secundaria, corroboran estos datos: entre 2007 y 2019, particularmente en estudiantes de secundaria, apreciamos indicadores que apuntan por una disminución en la lectura de libros y en la presencia / existencia de libros en el hogar. Por otro lado, solo el 12,1\% de los jóvenes en educación secundaria dice que lee libros con más frecuencia en dispositivos digitales; El 26,1\% lee noticias online; El 32,3\% investiga en la web sobre determinados temas; y el 20,4\% investigó aspectos prácticos.

El estudio de Mata y Neves (2020) señala algunas razones para los datos mencionados anteriormente. Así, destacamos que estos investigadores consideran que los cambios tecnológicos de la última década han hecho que los jóvenes pasen una cantidad de tiempo mucho mayor con los dispositivos digitales (smartphones, tablets, ordenadores), impulsados por un enorme incremento de los contenidos digitales y por el gran crecimiento y diversificación de redes sociales. Sin embargo, a pesar de este hallazgo, los datos presentados por estos

\footnotetext{
${ }^{1}$ Un consumo muy bajo, por parte de los jóvenes, de la prensa escrita tradicional (periódicos y revistas), sin que ello signifique, sin embargo, una transferencia estricta (y creciente) a los formatos de prensa digital, conclusión que también sitúa a las muestras trabajadas en el marco interno y transfronterizas; poca posibilidad de formar una clase de lectura (juvenil). (Traducción de los autores)
}

Espiral. Cuadernos del Profesorado | ISSN 1988-7701 | 2021, 14(29), 18-30 
investigadores muestran un bajo uso de lectura y escritura para los estudiantes de secundaria. De hecho, estos jóvenes pasan su tiempo en digital especialmente con la participación del chat online $(71.6 \%)$.

Por lo tanto, los resultados de estos estudios tienen implicaciones que preocupan para el desarrollo de la competencia lectora de los más jóvenes, como ya en 2009 lo refería Peter Hunt: "a precipitous decline in literacy among children, who are more used to fragmented reading patterns, and lack sustained commitment to (or training for) extended verbal narratives" (Hunt, 2009, p. 19).

El gran desafío para la sociedad nos parece hoy en día la formación de los más jóvenes para las alfabetizaciones múltiples, consiguiendo que el espacio digital también se considere un espacio de lectura, lectura de información y lectura literaria. La formación para la alfabetización visual, la alfabetización mediática, la alfabetización informacional, la alfabetización digital también es esencial, para comprender otros idiomas y diferentes discursos, para la capacidad de seleccionar, evaluar y utilizar la información y el contenido correctamente, para comunicarse de manera adecuada y segura. Todos estos requisitos contribuyen a la educación para la $e$ ciudadanía, mostrando a los jóvenes que el mundo digital es un espacio de comunicación, información, consumo, que debe ser inclusivo, abierto, plural, seguro y compartido por todos.

Así, en este texto, nuestro objetivo es centrarnos en el análisis de un conjunto de documentos que constituyen y contribuyen, entre otros, a la construcción del discurso pedagógico oficial en Portugal, en torno a lo digital. Como resultado de este análisis, reflexionamos sobre la presencia de esta dimensión específica en los textos oficiales, pero, sobre todo, sobre las posibles implicaciones de esa presencia.

\section{El discurso pedagógico oficial y digital}

Los documentos que, en el contexto de este artículo, consideramos serán los siguientes: Perfil de los alumnos a la salida la escolaridad obligatoria [Perfil dos Alunos à Saída da Escolaridade Obrigatória] (PASEO) ${ }^{2}$; Estrategia Nacional de Educación para la Ciudadanía [Estratégia Nacional de Educação para a Cidadania] (ENEC); documentos en el marco del Plan Nacional de Lectura 2027 [Plano Nacional de Leitura 2027] (PNL2027); documentos en el marco de la Red de Bibliotecas Escolares [Rede de Bibliotecas Escolares] (RBE). Es un conjunto de documentos que configuran el discurso oficial como "Official rules regulating the production, distribution, reproduction, interrelation, and change of legitimate pedagogic texts (discourse), their social relations of transmission and acquisition (practice), and the organization of their contexts (organization)"33 (Bernstein, 1990, p. 193). Este discurso se construye a través de un "recontextualising principle which selectively appropriates, relocates,

\footnotetext{
${ }^{2}$ Decidimos no destacar los documentos específicos para cada asignatura escolar, es decir, para la asignatura de Lengua Portuguesa. El análisis que se hace, en este contexto, de los documentos Aprendizajes Esenciales [Aprendizagens Essenciais] para todos los años de escolaridad (1. ${ }^{\circ}$ a $12 .^{\circ}$ año) - y los elegimos porque son los que ya están en línea con PASEO - revela que la referencia a lo digital es restringida, en todos los doce documentos, a una afirmación que, siendo significativa, solo se hace eco de la conceptualización formulada en PASEO: "uma participação segura nos «jogos de linguagem» que os falantes realizam ativando saberes de uma pluralidade de géneros textuais, em contextos que o digital tem vindo a ampliar." [una participación segura en los" juegos de lenguaje "que los hablantes realizan activando el conocimiento de una pluralidad de géneros textuales, en contextos que el digital viene expandiendo (Traducción de los autores)] (Aprendizagens Essenciais/Articulação com o Perfil dos Alunos, 2018, p. 2).

${ }^{3}$ Reglas oficiales que regulan la producción, distribución, reproducción, interrelación y cambio de textos pedagógicos legítimos (discurso), sus relaciones sociales de transmisión y adquisición (práctica) y la organización de sus contextos (organización). (Traducción de los autores)
}

Espiral. Cuadernos del Profesorado | ISSN 1988-7701 | 2021, 14(29), 18-30 
refocuses and relates other discourses to constitute its own order"4 . (Bernstein, 2000, p. 33). No analizaremos los textos más directamente relacionados con las asignaturas escolares, a saber, la asignatura de Lengua Portuguesa, ya que su mayor especificidad y focalización en contenidos particulares pierde algún alcance en el ámbito que se pretende analizar aquí, por un lado y, por otro, porque pretendemos solicitar documentos de mayor alcance y transversalidad. Dedicaremos un apartado a cada uno de los conjuntos de textos mencionados.

\section{Perfil de los alumnos a la salida de la educación obligatoria (PASEO)}

Este documento se estructura en torno a Principios y Visión (que deben orientar la acción educativa), Valores y Áreas de Conocimiento (a desarrollar). Pretende afirmarse, en su génesis y naturaleza, como un texto de gran alcance (basado en el supuesto del respeto al carácter inclusivo y multifacético de la Escuela), transversal (cada área curricular contribuye al desarrollo de todas las áreas de competencia) y recursivo (es posible que sus contenidos y finalidades puedan ser invocados en cualquier curso escolar). Se trata, por tanto, de un referente de gran proyección, de carácter no prescriptivo y que marca, de forma explícita, la matriz ideológica que se pretende en la formación de las generaciones futuras. Se asume como el documento que no puede ser eludido en el proceso de construcción social del currículo, apuntando a que es en la

educação escolar (...) que os alunos desta geração global constroem e sedimentam uma cultura científica e artística de base humanista. Para tal, mobilizam valores e competências que lhes permitem intervir na vida e na história dos indivíduos e das sociedades, tomar decisões livres e fundamentadas sobre questões naturais, sociais e éticas, e dispor de uma capacidade de participação cívica, ativa, consciente e responsável (Martins, 2017, p. 10) ${ }^{5}$

Esta matriz también se asume inscrita en el marco de documentos provenientes de la Unión Europea, OCDE y UNESCO. En este contexto, es sumamente significativo que, desde el prefacio, se incluyan los siete conocimientos necesarios para la Educación del Futuro, convocados por Edgar Morin:

prevenção do conhecimento contra o erro e a ilusão; ensino de métodos que permitam ver o contexto e o conjunto, em lugar do conhecimento fragmentado; o reconhecimento do elo indissolúvel entre unidade e diversidade da condição humana; aprendizagem duma identidade planetária considerando a humanidade como comunidade de destino; exigência de apontar o inesperado e o incerto como marcas do nosso tempo; educação para a compreensão mútua entre as pessoas, de pertenças e culturas diferentes; e desenvolvimento de uma ética do género humano, de acordo com uma cidadania inclusiva. (Martins, 2017, p. 6). ${ }^{6}$

\footnotetext{
${ }^{4}$ Principio recontextualizador que se apropia, reubica, reenfoca y relaciona selectivamente otros discursos para constituir su propio orden. (Traducción de los autores)

5 “educación escolar en la que los estudiantes de esta generación global construyan y establezcan una cultura científica y artística basada en el humanismo. Para ello, movilizan valores y habilidades que les permiten intervenir en la vida e historia de los individuos y sociedades, tomar decisiones libres e informadas sobre cuestiones naturales, sociales y éticas, y tener capacidad de participación cívica, activa, consciente y responsable.” (Traducción de los autores)

${ }^{6}$ Prevención del conocimiento contra el error y la ilusión; métodos de enseñanza que permitan ver el contexto y el conjunto, en lugar de conocimientos fragmentados; el reconocimiento del vínculo indisoluble entre unidad y diversidad de la condición humana; aprender una identidad planetaria considerando a la humanidad como una comunidad de destino; exigir señalar lo inesperado y lo incierto como marcas de nuestro tiempo; educación para
} 
En este ambicioso y desafiante proyecto educativo, la capacidad de comprender la naturaleza y las funciones de la tecnología, es fundamental. La aceleración de la Historia, la dinámica de la circulación del conocimiento y la información a escala planetaria, requieren la formación de ciudadanos capaces de actuar con / sobre la tecnología de manera crítica, potenciando el desarrollo de valores y competencias, en un mundo globalizado construido a partir de realidades locales, un proceso en el que la tecnología y, en este caso particular, las tecnologías de la información, son centrales, pero también asegurando que los medios no se conviertan, en gran medida, en fines. Si el fin último de la educación, el escolar aquí incluido, es permitir que todos descubran / construyan su propia humanidad, la tecnología debe estar al servicio de las personas y no al revés.

Así, se espera que, en este documento, se multipliquen las referencias de carácter más amplio a la tecnología, lo que ocurre, veintidós veces, en formulaciones que se enfocan en diferentes aspectos, aunque en relación entre sí: la evolución / desarrollo del conocimiento científico y tecnológico en el mundo actual; la necesidad de que la sostenibilidad surja del equilibrio entre los sistemas sociales, económicos y tecnológicos y el Sistema Tierra; el uso de las tecnologías de la información y la comunicación, como base para aprender y seguir aprendiendo a lo largo de la vida; el uso competente de diferentes lenguajes y símbolos asociados con las tecnologías, así como los idiomas, la literatura, la música y las artes; la capacidad de apreciar críticamente las realidades artísticas en diferentes soportes tecnológicos, integradas en sus contextos sociales, geográficos, históricos y políticos; trabajar utilizando materiales, instrumentos, herramientas, máquinas y equipos tecnológicos.

Destacamos dos momentos particulares de este documento: las secciones sobre sensibilidad estética y artística y saberes científicos y tecnológicos. Respecto al primero, se entiende como vital, en el proceso de desarrollo de la expresividad personal y social de los estudiantes, la existencia de "processos de experimentação, de interpretação e de fruição de diferentes realidades culturais." (Martins, 2017, p. 28) Entre las habilidades asociadas al desarrollo de la sensibilidad estética y artística, las más importantes son el reconocimiento de especificidades e intenciones de las diferentes manifestaciones culturales; la capacidad de experimentar la especificidad de la comunicación artística, la apreciación del papel de las diferentes formas de expresión artística en la vida de las comunidades humanas y la capacidad de "apreciar criticamente as realidades artísticas, em diferentes suportes tecnológicos, pelo contacto com os diversos universos culturais" (Martins, 2017, p. 28) Con respecto a los saberes científicos y tecnológicos, resaltamos:

manipular e manusear materiais e instrumentos diversificados para controlar, utilizar, transformar, imaginar e criar produtos e sistemas; adequar a ação de transformação e criação de produtos aos diferentes contextos naturais, tecnológicos e socioculturais, em atividades experimentais, projetos e aplicações práticas desenvolvidos em ambientes físicos e digitais (Martins, 2017, p. 29) ${ }^{9}$

\footnotetext{
el entendimiento mutuo entre personas, de diferentes orígenes y culturas; y desarrollo de una ética de la humanidad, de acuerdo con una ciudadanía inclusiva. (Traducción de los autores)

${ }^{7}$ Procesos de experimentación, interpretación y disfrute de diferentes realidades culturales. (Traducción de los autores)

${ }^{8}$ Valorar críticamente las realidades artísticas, en diferentes soportes tecnológicos, a través del contacto con los diferentes universos culturales. (Traducción de los autores)

${ }^{9}$ Manipular y manejar diversos materiales e instrumentos para controlar, usar, transformar, imaginar y crear productos y sistemas; adaptar la acción de transformación y creación de productos a diferentes contextos naturales,
} 
Es relevante que el documento destaque que "Os alunos percebem o valor estético das experimentações e criações a partir de intencionalidades artísticas e tecnológicas, mobilizando técnicas e recursos de acordo com diferentes finalidades e contextos socioculturais."10. (Martins, 2017, p. 28)

También importante en el apartado lenguage y textos es la afirmación de que los estudiantes deben ser capaces de utilizar códigos de forma eficaz que permitan "exprimir e representar conhecimento em várias áreas do saber, conduzindo a produtos linguísticos, musicais, artísticos, tecnológicos, matemáticos e científicos" ${ }^{11}$ (Martins, 2017, p. 21), que debe aplicarse a diferentes contextos comunicativos, en un entorno analógico y digital. Los estudiantes asimismo deben ser capaces de "dominar capacidades nucleares de compreensão e de expressão nas modalidades oral, escrita, visual e multimodal.""12 (Martins, 2017, p. 21)

En resumen, PASEO es un documento que apunta a pautas como la formación de individuos para una ciudadanía activa e informada, para la formación a lo largo de la vida, para el dominio de alfabetizaciones múltiples. El dominio de la Tecnología y lo digital, en una perspectiva crítica, se considera fundamental para todo el proceso de enseñanza y aprendizaje y para la integración del individuo en una sociedad donde se debe priorizar la construcción de valores humanísticos.

\section{Estrategia Nacional de Educación para la Ciudadanía (ENEC)}

La ENEC apunta al desarrollo de la formación, en el sistema educativo portugués, de las personas que se constituyen en ciudadanos activos y conscientes en la sociedad contemporánea, en tiempos de grandes desafíos y demandas. Este documento pone en el centro temas como la sostenibilidad, la interculturalidad, la inclusión, la igualdad, la identidad y la alteridad. Todos estos temas, de gran relevancia en la sociedad y en la escuela actual, deben ser abordados por los docentes.

La escuela se ve aquí como el contexto donde los estudiantes adquieren las habilidades que les permiten conocer y reflexionar sobre estos mismos temas estructurantes. Entre estas habilidades, destacamos las múltiples alfabetizaciones que naturalmente permiten a los estudiantes trabajar con la lectura y la escritura, pero también con la alfabetización informacional, la alfabetización mediática y la alfabetización digital. El dominio de las alfabetizaciones múltiples permitirá a docentes y alumnos hacer un trabajo más enriquecedor en la promoción de la educación para la ciudadanía, ya que el documento recomienda un enfoque integrado en el currículo, pero basado en la vida cotidiana de la escuela y en articulación con la comunidad. En este enfoque se privilegian las metodologías activas y los procesos experienciales, donde los estudiantes realmente pueden adquirir y desarrollar habilidades personales y sociales.

Esta estrategia, plasmada en el documento mencionado, tiene cuidado de identificar qué competencias deben poseer los docentes que trabajan en el tema de la educación para la ciudadanía en la escuela. Así, entre otros, el docente "Deve possuir competências de utilização

\footnotetext{
tecnológicos y socioculturales, en actividades experimentales, proyectos y aplicaciones prácticas desarrolladas en entornos físicos y digitales. (Traducción de los autores)

${ }^{10}$ Los estudiantes perciben el valor estético de los experimentos y creaciones desde intenciones artísticas y tecnológicas, movilizando técnicas y recursos de acuerdo con diferentes propósitos y contextos socioculturales. (Traducción de los autores)

${ }^{11}$ Expresar y representar conocimientos en diversas áreas del conocimiento, dando lugar a productos lingüísticos, musicales, artísticos, tecnológicos, matemáticos y científicos. (Traducción de los autores)

${ }^{12}$ Dominar las habilidades básicas de comprensión y expresión en los modos oral, escrito, visual y multimodal. (Traducción de los autores)
} 
de meios tecnológicos e de Plataformas Digitais; Deve possuir competências de utilização de meios tecnológicos" 13 (Monteiro, 2017, p. 14).

La necesidad de que los profesores dominen estas alfabetizaciones múltiples se debe en última instancia a que los recursos para trabajar con la educación para la ciudadanía en la escuela están en línea, en el sitio web de la Dirección General de Educación (https: //www.dge.mec.pt/areas-tematicas). El trabajo con alfabetizaciones múltiples y su desarrollo en los estudiantes facilitará la creación y difusión de sus proyectos en páginas web, blogs, facebook u otras plataformas digitales de audio y vídeo.

Parece fundamental mencionar, en una estrategia nacional de educación ciudadana, el tema de la ciudadanía digital o e-ciudadanía. El documento de ENEC no hace referencia explícita al término ciudadanía digital, pero nos parece que podemos decir que subyace en este marco. Teniendo en cuenta el marco Digital Citizenship Defined (Ribble et al., 2016), desarrollado en la Universidad de Kentucky, en los Estados Unidos de América, la ciudadanía digital se entiende como un comportamiento adecuado y participación responsable en el uso de la tecnología por parte de los ciudadanos. Según Ribble et al. (2016, p. 4)

the idea was to create a framework of defining elements that provide a structure for digital citizenship education on which everything else could hang. (...) As a result, we identified nine key elements that help define how to best use technology in every school, home and community. They're organized into three primary categories: respect, educate, protect. $^{14}$

Estas tres categorías -respeto, educación, protección- definen los distintos elementos que componen la ciudadanía digital, como la alfabetización digital, la comunicación digital o la salud y el bienestar digital, alertando de los riesgos y el equilibrio necesario. en el uso de lo digital, ya que "Digital citizenship is a complex topic with many facets. We need to make sure we help students understand the issues that might occur online while also stressing the positive impact of technology"15 (Ribble et al., 2016, p. 4).

En resumen, la estrategia de educación ciudadana tiene la misión de formar personas que sean ciudadanos capaces de intervenir activamente en sus comunidades, dotados de competencias personales y sociales, y con un alto dominio de alfabetizaciones múltiples, lo que sin duda es una condición fundamental para su desarrollo y desempeño en la sociedad.

\section{El ámbito del Plan Nacional de Lectura 2027 (PNL2027)}

El PNL2027 en sí mismo es una herramienta digital; estamos ante un sitio web donde no solo se encuentra la legislación que creó el PNL2027, sino también su marco estratégico (Quadro Estratégico PNL2027).

Además de estos documentos, en este sitio web los docentes tienen acceso a un enorme conjunto de estudios, acciones, proyectos y un amplio abanico de recursos, tanto a nivel nacional como internacional, que hacen del conocimiento, análisis y reflexión acerca de esta

\footnotetext{
${ }^{13}$ Debe tener habilidades en el uso de medios tecnológicos y plataformas digitales; Debe tener habilidades en el uso de medios tecnológicos. (Traducción de los autores)

${ }^{14}$ La idea era crear un marco de elementos definitorios que proporcionaran una estructura para la educación para la ciudadanía digital en la que todo lo demás pudiera depender. (...) Como resultado, identificamos nueve elementos clave que ayudan a definir la mejor manera de utilizar la tecnología en cada escuela, hogar y comunidad. Están organizados en tres categorías principales: respetar, educar, proteger. (Traducción de los autores)

${ }^{15}$ La ciudadanía digital es un tema complejo con muchas facetas. Necesitamos asegurarnos de ayudar a los estudiantes a comprender los problemas que pueden ocurrir en línea y, al mismo tiempo, enfatizar el impacto positivo de la tecnología. (Traducción de los autores)
} 
página web un desafío, en el sentido de poder capitalizarla y movilizarla eficazmente para la práctica pedagógica y didáctica de los docentes.

El PNL2027 también contiene el Catálogo PNL2027 en su sitio web. Este Catálogo constituye un recurso fundamental para los docentes de todos los niveles educativos. Creado en colaboración con las Bibliotecas Municipais de Lisboa, aquí encontramos una amplia gama de títulos recomendados para la lectura recreativa, pero también para la lectura en el aula. El Catálogo es tanto más importante cuanto que los documentos curriculares oficiales para la educación preescolar y para la educación primaria no contienen sugerencias de obras para ser leídas, en el contexto escolar, con los estudiantes; por tanto, es a través de esta herramienta que los profesores pueden elegir qué libros consideran más adecuados para leer, compartir y trabajar con sus alumnos.

Entonces, podemos considerar que el PNL2027 prácticamente no tiene existencia física, pero sobre todo una existencia digital.

Para una mejor comprensión y análisis de la forma en que lo digital pasa por el PNL2027, nos enfocamos en la legislación que lo impulsa y su Quadro Estratégico. En la Resolução do Conselho de Ministros n..$^{\circ}$ 48-D/2017, el Gobierno de Portugal aprueba las directrices para el PNL2027. Entre ellos destacamos las líneas:

b) Lançar programas dirigidos a crianças, jovens e adultos, que visem promover o desenvolvimento de literacias múltiplas, designadamente, a da leitura e escrita, a digital, da informação visual, científica e tecnológica, por forma a preparar a população portuguesa para as exigências da sociedade do século XXI;

f) Promover as relações entre a leitura, a literatura, as artes, as ciências e a tecnologia e fomentar a cultura científica, tecnológica e artística, em colaboração com instituições de ciência e de cultura; (RCM, 2017, p. 6). ${ }^{16}$

Por tanto, la legislación que promueve el PNL2027 ofrece claras indicaciones del público-objetivo cubierto, que básicamente consiste en toda la población portuguesa, niños, jóvenes y adultos, en un intento de continuar la formación, iniciada en la década anterior en la primera fase de lo Plan Nacional de Lectura, para lectura, escritura y alfabetizaciones múltiples. Con el objetivo de capacitar a la población portuguesa para una sociedad cada vez más exigente y desafiante, las alfabetizaciones múltiples integran la alfabetización digital no como un fin en sí mismo, sino en una relación que se debe promover entre lectura, literatura y tecnología, entre otros.

Estas desafiantes pautas se actualizan en el Quadro Estratégico. Este documento establece los objetivos a promover, de los que destacamos "Potenciar a presença e a projeção mediática da leitura nos meios escritos, impressos e digitais, em presença e na Internet” y "Associar a leitura às ciências, às humanidades, às artes e às tecnologias digitais, de acordo com uma nova ecologia que se faz de múltiplas literacias"17 (PNL2027, 2017, p. 13).

\footnotetext{
${ }^{16}$ b) Lanzar programas dirigidos a niños, jóvenes y adultos, que tengan como objetivo promover el desarrollo de alfabetizaciones múltiples, a saber, lectura y escritura, información digital, visual, científica y tecnológica, con el fin de preparar a la población portuguesa para las demandas. Sociedad del siglo XXI; f) Promover la relación entre lectura, literatura, artes, ciencias y tecnología y promover la cultura científica, tecnológica y artística, en colaboración con instituciones científicas y culturales. (Traducción de los autores)

${ }^{17}$ Potenciar la presencia y proyección mediática de la lectura en medios escritos, impresos y digitales, en presencia y en Internet y Asociar la lectura con las ciencias, humanidades, artes y tecnologías digitales, de acuerdo con una nueva ecología que se está haciendo de alfabetizaciones múltiples. (Traducción de los autores)
} 
Estos objetivos se plasman y recontextualizan en la escuela, en las aulas y en las bibliotecas escolares a través de las siguientes Áreas de Intervención, también presentes en el Quadro Estratégico: "Valorização de todas as literacias; Aproximação à literatura, ciências, artes e tecnologias; Exploração da Web como espaço de partilha, difusão e comunicação" (PNL2027, 2017, p. 19). ${ }^{18}$

De esta forma, el espacio digital está muy presente en el PNL2027, en primer lugar, en asumir la misma importancia para todas las alfabetizaciones, ya que todas ellas son valoradas. La valoración de todas las alfabetizaciones tiene en cuenta que la lectura y la escritura son competencias transversales y fundamentales clave para que las personas se muevan con soltura y eficacia en diferentes contextos informativos y comunicacionales. En el Quadro Estratégico incluso se afirma que estamos formando un "nuevo sujeto lector" (PNL2027, 2017, p. 23), que en palabras de Mendoza (2012) deberá ser capaz de gestionar su actividad cognitiva, de crear redes y conexiones, en cualquier espacio de lectura, es decir, en el espacio digital. Mendoza (2012) afirma que el espacio de lectura digital se presenta al lector como atractivo, lo invita a nuevos desafíos y le obliga a saber leer todo tipo de hipertextos y leer de forma no lineal; este espacio de lectura digital obliga al lector a leer sin perderse en las múltiples vías de acceso a los hipertextos que se abren en el espacio digital.

Además de esta relación entre la lectura y la escritura con el espacio digital, el nuevo lector también debe dominar otras formas de lectura relacionadas con el audio, vídeo, visual, informativo.

La valorización de todas estas alfabetizaciones permitirá un acercamiento a varias áreas del conocimiento. Estas habilidades transversales clave permiten al individuo tener una lectura mucho más rica y comprensiva del mundo, donde la literatura y las humanidades, el arte, la ciencia y la tecnología exigen creatividad, pensamiento crítico y divergente, experiencia estética.

La tecnología, ya sea de audio, vídeo, digital, multimedia, se convierte en uno de los principales objetivos de este PNL2027. Se incentivan fuertemente los proyectos y actividades de lectura y escritura, en una apelación al uso de herramientas digitales que apuestan por la creación en la web e incluso por la creación de carácter literario. Promover la publicación de diferentes textos de los estudiantes en blogs o páginas de facebook, fomentar la escritura a través de fanfics o storytelling, leer literatura online, participar en clubes virtuales de lectura y escritura son formas de entender la exigencia de un dominio completo de las habilidades de leer y escribir. La tecnología se convierte entonces en una herramienta que posibilita la relación e integración con la literatura y el arte.

La apuesta por el espacio digital para la lectura y la escritura lleva al PNL2027 a considerar la web como su espacio privilegiado para compartir y comunicarse. Esta existencia fundamentalmente digital (como ya hemos mencionado) fomenta la reflexión de la escuela, el profesorado, la sociedad en general, no solo sobre una nueva formación de lectores sino también sobre la alfabetización y la ciudadanía digital, cuyas competencias, fundamentales para que las personas se muevan en la sociedad digital, deben ser promovidas, enseñadas, aprendidas y comprendidas por todos.

En resumen, el PNL2027 favorece la promoción de la lectura, la escritura, la alfabetización múltiple; También se privilegia el espacio digital, que tiene principalmente una función instrumental. Sin embargo, se aprecia, un cierto atractivo hacia la creación e incluso a

\footnotetext{
${ }^{18}$ Valoración de todas las alfabetizaciones; Aproximación a la literatura, las ciencias, las artes y las tecnologías; Exploración de la Web como espacio de intercambio, difusión y comunicación. (Traducción de los autores)
}

Espiral. Cuadernos del Profesorado | ISSN 1988-7701 | 2021, 14(29), 18-30 
la creación de carácter literario, ya que se invita y anima a los alumnos a escribir y publicar textos en la web que se pueden configurar como una iniciación a una obra de carácter más literario.

\section{Red de Bibliotecas Escolares}

La Red de Bibliotecas Escolares (RBE), con una amplia implementación en las escuelas de todo el país, es una estructura física, compuesta por un conjunto de bibliotecas escolares que integran completamente estas escuelas. No obstante, RBE también tiene una fuerte presencia en el mundo digital a través de su página web donde se encuentran toda la legislación y referencias teóricas que rigen esta estructura, así como estudios, recursos, proyectos, posibilidades de formación, en una mirada, que se requiere atenta, por modelos y experiencias nacionales e internacionales. La RBE y la biblioteca escolar de cada escuela constituyen así un desafío complejo y atractivo tanto para el equipo bibliotecario escolar, coordinado por el docente-bibliotecario, como para todos los docentes y demás comunidad educativa, con la expectativa de un trabajo colaborativo entre todas.

Los marcos teóricos que rigen la RBE otorgan gran importancia a lo digital. Para comprender mejor este aspecto, veremos los documentos Programa Rede de Bibliotecas Escolares. Quadro Estratégico: 2014-2020 (RBE, 2013) y Aprender com a Biblioteca Escolar (Conde, Mendinhos \& Correia, 2017), cuya 1. ${ }^{\mathrm{a}}$ edición se remonta a 2012, y posteriormente se amplió en el 2017.

El documento Quadro Estratégico (RBE, 2013) tiene la misión de ser una guía para la actividad de todas las bibliotecas escolares y ser un instrumento de referencia que permita mejorar los planes de acción de estas mismas bibliotecas. Por tanto, define un conjunto de prioridades, de dimensiones clave para todo el desempeño de las bibliotecas escolares. En estas dimensiones clave, se destacan la promoción de la lectura y alfabetizaciones múltiples, alfabetización digital, alfabetización informacional, alfabetización mediática:

3. Locais de formação e desenvolvimento da competência leitora, condição de todo o conhecimento. 4. Focos difusores do gosto e do prazer de ler, fundamentais à construção de hábitos de leitura. 5. Áreas de ensino, essenciais à formação para as literacias digitais, dos média e da informação. 7. Ambientes flexíveis, adaptados às mudanças tecnológicas e às necessidades dos utilizadores. (RBE, 2013, p. 9) ${ }^{19}$

La competencia lectora, el gusto y el placer por la lectura son de hecho áreas privilegiadas, considerando que el desarrollo de la competencia lectora es fundamental. Solo la formación de lectores competentes permitirá la adquisición y el aprendizaje en cualquier área del conocimiento, posibilitando así la exploración y convivencia con estrategias, herramientas, recursos tecnológicos y digitales. En este documento se asume que este es un rol de la biblioteca escolar, que tiene como misión formar lectores capaces de un dominio literario para acceder a la construcción personal, el éxito escolar y la intervención basada en la sociedad.

Por otro lado, y en estrecha relación con la competencia lectora, el documento llama la atención sobre la Tecnología, el espacio digital y el dominio de alfabetizaciones múltiples. La preocupación se centra en la formación del estudiante para la investigación, para la selección y evaluación de la información, para su uso pero también para la producción y comunicación de

\footnotetext{
193 Lugares de formación y desarrollo de la competencia lectora, condición de todo conocimiento. 4 Enfoques que difunden el gusto y el placer de la lectura, fundamentales para la construcción de hábitos lectores. 5 Áreas de enseñanza, fundamentales para la formación de alfabetizaciones digitales, mediáticas e informacionales. 7 Entornos flexibles, adaptados a los cambios tecnológicos y las necesidades de los usuarios. (Traducción de los autores)
}

Espiral. Cuadernos del Profesorado | ISSN 1988-7701 | 2021, 14(29), 18-30 
esta misma información. La formación del ciudadano digital o para la e-ciudadanía se refleja en este documento, donde se llama la atención sobre el crecimiento de la información en el mundo digital, la necesidad de una participación segura e informada en el mundo de la web y la realidad de un acceso a este mundo que tiene que ser flexible e inclusivo.

El documento Aprender com a Biblioteca Escolar (Conde, Mendinhos \& Correia, 2017) también constituye un referente para la acción desarrollada no solo en las bibliotecas escolares, sino también en la escuela, en una llamada de atención a la necesidad de un trabajo colaborativo real y efectivo en la escuela y la comunidad educativa. Tres áreas de anclaje están presentes en este marco: competencia lectora, alfabetización mediática y alfabetización informacional.

Naturalmente, existe una relación muy estrecha entre los dos documentos que constituyen referencias para la biblioteca escolar. Sin embargo, este marco tiene algunas particularidades. En primer lugar, hay una preocupación muy explícita por cubrir toda la escolaridad en Portugal, desde la educación preescolar hasta la secundaria. Por otro lado, para cada una de las alfabetizaciones mencionadas y para cada nivel educativo, se comparten diferentes estrategias de trabajo que buscan asistir y orientar la acción de la biblioteca escolar y la escuela, en un trabajo colaborativo cercano.

En estas estrategias de trabajo, para todas las alfabetizaciones mencionadas, lo digital marca una presencia muy fuerte, tanto a nivel de lectura como a nivel de escritura, en la comprensión de la relación fundamental y complementaria entre comprensión y producción de código escrito, como afirma Santana (2009, p. 28) "os processos de escrita e de leitura, inscritos em situações relacionais, constituem permanentes negociações de significado, tendo sempre presente o outro para quem se escreve e de quem se lê, reconstruindo internamente o sentido"20.

Así, leer libros electrónicos, participar en clubes de lectura virtuales, usar y leer en bibliotecas virtuales, construir blogs, producir booktrailers de libros o storytellings son solo algunas actividades sugeridas que nos llevan a leer y producir textos.

En resumen, las referencias para la biblioteca escolar, sin olvidar las bibliotecas escolares que existen en las escuelas portuguesas, bajo la tutela de RBE, enfatizan el espacio digital como un lugar (ámbito) que promueve la alfabetización múltiple, y apuntan claramente a la necesidad del trabajo colaborativo entre equipo de biblioteca escolar, liderado por el docente-bibliotecario, y toda la comunidad educativa.

\section{Conclusión}

Los documentos analizados destacan una realidad en la que se espera la formación de los futuros ciudadanos a partir de supuestos humanistas, de matriz contemporánea, teniendo en cuenta las especificidades del mundo actual y, en esa medida, previendo una fuerte presencia de lo digital, articulado con una marcada preocupación por la educación de un ciudadano insertado en su realidad local, pero construyendo sostenibilidad a escala global. En este proceso, la capacidad crítica es una cualidad destacada. Los estudiantes deben aprender a utilizar diferentes soportes, distintos recursos tecnológicos, movilizarlos en diferentes situaciones y con diferentes propósitos, pero siempre de manera crítica; el filtro crítico vendrá de la matriz humanista. En un mundo caracterizado por el avance tecnológico, es necesario que se configure como potenciador, como facilitador y, en esa medida, se integre de forma natural en la vida cotidiana de los procesos educativos.

\footnotetext{
${ }^{20}$ Los procesos de escritura y lectura, inscritos en situaciones relacionales, constituyen negociaciones permanentes de significado, teniendo siempre presente al otro para quien escribe y para quien lee, reconstruyendo internamente el significado. (Traducciõn de los autores)
}

Espiral. Cuadernos del Profesorado | ISSN 1988-7701 | 2021, 14(29), 18-30 
Así, se aprecia una gran presencia de lo digital en estos documentos, siempre con una dimensión instrumental, la que parece ser la apropiada. Un claro ejemplo es el propio PNL2027, sobre lo que podemos reforzar que es exclusivamente digital en la forma en que se da a conocer y está disponible para su posterior funcionamiento. Asimismo, es significativo que, a partir de los distintos documentos analizados, veamos emerger el concepto de ciudadanía digital.

La importancia de lo digital en la creación surge, de manera explícita, como una de las dimensiones instrumentales fundamentales en la respuesta emancipadora de quienes pasan por la escuela y, en este camino de doce años, hagan un fuerte compromiso con el futuro. La creación artística y literaria mencionada aquí, emerge solo de manera tenue. Sin embargo, esta dimensión se engloba, tanto en la atención que los documentos otorgan a la importancia, en la época contemporánea, de un perfil humanista en el que la creatividad juega un papel fundamental, como en la relevancia que lo digital asume, de manera ineludible, en este proceso. Por ejemplo, en este sentido, se puede leer en PASEO que "Perante os outros e a diversidade do mundo, a mudança e a incerteza, importa criar condições de equilíbrio entre o conhecimento, a compreensão, a criatividade e o sentido crítico." (Martins, 2017, p.5) ${ }^{21}$

Particularmente relevante es el grado de congruencia de los diferentes conjuntos de textos que aquí hemos convocado, ya sea PASEO, en conjunto con Aprendizagens Essenciais para las distintas asignaturas, ya sea la ENEC integral, o los documentos que resultan del papel sumamente relevante que juega la actividad del PNL2027, especialmente en su más reciente reconfiguración, ya sean las resultantes del trabajo realizado en el ámbito de la RBE, con una importante presencia en la vida cotidiana de las escuelas.

Lo digital, en su instrumentalidad, es una herramienta imprescindible para que se lleven a cabo las funciones de la escuela y otras instancias de la sociedad. En la educación de los estudiantes, la creación debe asumir un papel central: "O processo da criação e da inovação tem de ser visto relativamente ao poeta, ao artista, ao artesão, ao cientista, ao desportista, ao técnico - em suma à pessoa concreta que todos somos" (Martins, 2017, p.6) ${ }^{22}$. El desafío que se plantea a los docentes, estudiantes y demás agentes educativos es que la creación no se pierda en la traducción de estos documentos oficiales en prácticas y que lo digital, esencial en su carácter instrumental, no adquiera esencialidad en los procesos.

Contribución de cada Autor: Introducción, Ângela Balça y Paulo Costa; El discurso pedagógico oficial y digital, Ângela Balça y Paulo Costa; Conclusión, Ângela Balça y Paulo Costa; escritura, revisión y edición, Ângela Balça y Paulo Costa.

Financiación: Esta investigación no recibió ninguna financiación externa.

Conflicto de Intereses: Los autores declaran que no tienen conflicto de intereses.

\section{Referencias}
Aprendizagens Essenciais/Articulação com $\quad$ o $\quad$ Perfil dos Alunos. (2018). https://www.dge.mec.pt/sites/default/files/Curriculo/Aprendizagens_Essenciais/1_ciclo/ae_1.o_ano_1o _ciclo_eb_portugues.pdf

Bernstein, B. (1990). The structuring of pedagogic discourse. Volume IV. Class, codes and control. Routledge.

Bernstein, B. (2000). Pedagogy, Symbolic Control and Identity. Theory, Research, Critique. Rowman \& Littlefield.

\footnotetext{
${ }^{21}$ Ante los demás y la diversidad del mundo, el cambio y la incertidumbre, es importante crear condiciones de equilibrio entre conocimiento, comprensión, creatividad y sentido crítico. (Tradición de los autores)

${ }^{22}$ El proceso de creación e innovación hay que verlo en relación con el poeta, el artista, el artesano, el científico, el deportista, el técnico, en definitiva, la persona concreta que todos somos. (Traducción de los autores)
} 
Conde, E., Mendinhos, I. \& Correia, P. (2017). Aprender com a biblioteca escolar (2. ${ }^{\text {a }}$ ed.). RBE. https://rbe.mec.pt/np4/np4/?newsId=1906\&fileName=referencial_2017.pdf

Hunt, P. (2009). The decline and decline of literary literacy: childhood and children's literature in the UK today. In A. M. Ramos, A. Prole, F. L. Viana, J. P. Cotrim, S. R. Silva (Orgs.), Formar leitores para ler o mundo (pp. 19-31). Fundação Calouste Gulbenkian.

Martins, G. (Coord.) (2017) Perfil dos Alunos à Saída da Escolaridade Obrigatória. Lisboa: Editorial do Ministério da Educação Ciência. https://www.dge.mec.pt/sites/default/files/Curriculo/Projeto_Autonomia_e_Flexibilidade/perfil_dos_alu nos.pdf

Mata, J. T. \& Neves, J. S. (Coords.) (2020, setembro 30). Práticas de Leitura dos Estudantes dos Ensinos Básico e Secundário - Primeiros resultados [Comunicação apresentada]. Apresentação do estudo, Lisboa, ISCTE. http://www.pnl2027.gov.pt/np4/file/1940/PLEP_Apresenta_o_p_blica_30_9_2020.pdf

Mendoza, A. (2012). Presentación. In A. Mendoza (Coord.), Leer hipertextos. Del marco hipertextual a la formación del lector literario (pp. 9-32). Octaedro.

Monteiro, R. (Coord.). (2017). Estratégia Nacional de Educação para a Cidadania. Lisboa: Ministério da Educação / Secretaria de Estado para a Cidadania e Igualdade. https://www.dge.mec.pt/sites/default/files/Projetos_Curriculares/Aprendizagens_Essenciais/estrategia_c idadania_original.pdf

Morgado, M. (2019). Literacia e conectividade intercultural: práticas de leitura para jovens. Álabe, 19, 1-17. https://doi.org/10.15645/Alabe2019.19.1

PNL2027. (2017). Quadro Estratégico Plano Nacional de Leitura 2027. http://pnl2027.gov.pt/np4EN/file/8/QE.pdf

RBE. (2013). Programa Rede de Bibliotecas Escolares. Quadro estratégico: 2014-2020. https://www.rbe.mec.pt/np4/np4/?newsId=1048\&fileName=978_972_742_366_8.pdf

Resolução do Conselho de Ministros (RCM) n. ${ }^{\circ}$ 48-D/2017 (2017). Diário da República: I Série, n. ${ }^{\circ}$ 65/2017, 1 . $^{\circ}$ Suplemento. https://dre.pt/application/conteudo/106816250

Rosário, C. (2021, no prelo). A relação de uma população estudantil com as bibliotecas num distrito português: resultados de duas pesquisas extensivas (2004-2013). In J. S. Pimenta, A. Balça, M. F. Silva (Orgs.), Biblioteca escolar, livros, leitura: interações e diálogos. Edufro.

Santana, I. (2009). Iniciação e desenvolvimento da escrita - dois percursos. Escola Moderna, 33, 25-40. 